\title{
TRANSPORTE AEROMÉDICO EM ASA FIXA E ROTATIVA
}

\author{
Bruno Gonçalves da Silva ${ }^{1}$, André Alves Elias ${ }^{2}$, Maria Eduarda Becho Arger Mar- \\ chetti $^{3}$, Vânia Paula de Carvalho ${ }^{4}$
}

\section{RESUMO}

O transporte de pacientes é um recurso com histórico evolutivo, iniciado no século XVIII, por métodos rudimentares como carruagens. Com a evolução tecnológica, foram incorporadas a este serviço médico equipes treinadas para utilização de inúmeras técnicas desenvolvidas para veículos adaptados como aviões e helicópteros. $\mathrm{O}$ transporte pode ser divido entre intra-hospitalar (entre setores de um hospital) e inter-hospitalar (entre hospitais diferentes). Propõe-se aqui um estudo da revisão de literatura crítica com o objetivo de descrever algumas características do transporte aeromédico inter-hospitalar de neonatos e crianças; as vantagens e desvantagens para a utilização de aeronaves de asas rotativas e fixas; apresentar as legislações que regulamentam essa modalidade de remoção tanto pela Portaria no 2048/GM quanto por exigência dos órgãos regulamentadores da aviação brasileira. Dessa forma, o estudo mostra que há necessidade de avaliar alguns critérios básicos para melhor escolha do tipo de aeronave para cada situação e faixa etária do doente.

Palavras-chaves: resgate aeromédico, enfermagem, uti neonatal, uti pediátrica.

\section{INTRODUÇÃO}

O transporte de pacientes é um recurso com histórico evolutivo, que teve início no século XVIII, quando ainda eram utilizados métodos rudimentares como carruagens para transferir um enfermo até um local onde houvesse uma pessoa para prestar alguma assistência. Com a evolução tecnológica e da medicina, foi possível adaptar veículos para transferir pacientes com segurança e com devidos critérios básicos de transportes emergenciais e não emergenciais. Aqueles que se caracterizam como emergenciais contam com uma equipe treinada para utilização de inúmeras técnicas desenvolvidas para veículos adaptados tais como ambulâncias, aviões, helicópteros entre outros (LACERDA, CRUVINEL, SILVA, 2011; MARTINEZ, GARCIA, CASARRUBIAS, 2011; GRAÇA, et al, 2017).

\footnotetext{
1. Enfermeiro de bordo da Unimed Aeromédica. Doutorando Tecnologia da Informação e Gestão do Conhecimento, pela FUMEC - BH. Docente da Faculdade de Ciências Médicas de Minas Gerais. Especialista em Terapia Intensiva, pediátrica, neonatal e adulto. E-mail: brunoenf87@gmail.com

2. Médico de Bordo da Unimed Aeromédica. Médico do Batalhão de Operações Aéreas do Corpo de Bombeiros Militar de Minas Gerais. Médico do SAMU 192 de Belo Horizonte. Contato: andreelias2601@gmail.com

3. Médica de Bordo da Unimed Aeromédica, Anestesiologista do Hospital das Clínicas da Universidade Federal de Minas Gerais - UFMG. Contato: eduardarger@gmail.com

4. Enfermeira de Bordo e Coordenadora de Enfermagem (RT) da Unimed Aeromédica BH. Mestre e pesquisadora do Grupo de Pesquisas SAUVI Trânsito. Docente da Faculdade de Ciências Médicas de Minas Gerais e Docente convidada do IEC- PUCMG. E-mail: vaniapaula.carvalho@gmail.com
} 
Por questões éticas, está a cargo do médico assistente ao enfermo, a responsabilidade de avaliar, diagnosticar a patologia e verificar as possíveis intervenções. Cabe a esse médico também identificar que a transferência do paciente é a opção mais viável no momento, levando em consideração os critérios de risco versus benefícios, e estabilizar o paciente para iniciar a remoção propiciando estabilidade respiratória e ventilatória. (MARTINEZ, et al, 2011).

A remoção de paciente é um recurso utilizado quando um enfermo se encontra em um local que possui limitações terapêuticas. Por isso surge a necessidade de transferência para outra instituição que possua recursos necessários para execução da terapêutica correta para o diagnóstico previamente identificado. Por essa razão adaptaram-se veículos, tornando-os apropriados para realizar o transporte com o máximo de segurança. Surge então, neste estudo, a iniciativa de descrever sobre os transportes neonatais e pediátricos por meio do serviço aeromédico.

\section{METODOLOGIA}

Este estudo apresenta uma revisão de literatura. A busca bibliográfica foi realizada no período compreendido entre maio e julho de 2019. As bases de dados escolhidas por este estudo foram as seguintes: BVS (Biblioteca Virtual em Saúde) e Scielo. Para a busca dos artigos científicos, utilizaram-se os seguintes descritores: "resgate aeromédico" E "enfermagem" E "uti neonatal" E "uti pediátrica" em cada uma das bases de dados citadas. Os critérios de inclusão foram (i) artigos publicados nos últimos dez anos, (ii) artigos publicados nos idiomas português, inglês e espanhol. Outros artigos foram identificados a partir das listas de referências bibliográficas citadas nos artigos selecionados neste estudo.

\section{RESULTADOS E DISCUSSÕES}

O transporte de paciente deve ser uma decisão na qual são avaliados os benefícios versus os riscos em escala potencial. Faz-se necessário considerar as terapias e/ou diagnósticos que não são possíveis no hospital de origem. Dentre as modalidades de transporte existentes, destacam-se a intra-hospitalar que se transfere um paciente entre setores da mesma instituição, e a inter-hospitalar que trata da remoção de um paciente para outra instituição (JUNIOR, NUNES, FILHO, 2010).

Para que haja o transporte inter-hospitalar, deve-se viabilizar, inicialmente, a vaga no hospital de destino, iniciando-se pelo contato prévio do médico de origem 
com médico da instituição que se tentará vaga. No caso de exames diagnósticos, a possibilidade de definir mais precisamente o horário e protocolos para realização do procedimento. Após se ter uma confirmação do serviço ou da vaga, deve se, então, viabilizar o meio que será transportado o paciente em questão (TRAIBER, ANDREOLIO, LUCHESE, 2006).

Para a escolha do modo de transporte, há fatores a serem levados em consideração, tais como: distância e duração, urgência da situação, complicações possíveis que o paciente possa evoluir durante um transporte aéreo ou terrestre. Além disso, devem ser verificados os fatores específicos de cada região ou meteorológicas (MANNARINO, TIMERMAN, 1998).

O transporte aeromédico exige uma junção de recursos materiais, medicamentos, dispositivos e equipe de um treinamento eficaz para a equipe que atuará no transporte de pacientes, uma vez que os profissionais de saúde serão contados como tripulantes e sujeitos a aprovação e treinamento pelo Departamento de Aviação Responsável.

Portanto, o meio aéreo é o mais utilizado para percorrer grandes distâncias em pouco tempo, além de permitir o acesso a áreas mais remotas para um resgate quando utilizada uma aeronave de asas móveis, por exemplo (LACERDA, CRUVINEL, SILVA, 2011).

A aeronave de asa rotativa possui uma autonomia média de até $400 \mathrm{~km}$ consideravelmente, sendo sua velocidade entre $200 \mathrm{~km} / \mathrm{h}$ a $320 \mathrm{~km} / \mathrm{h}$ dependendo do modelo da aeronave. Esse tipo de aeronave também possui múltiplas funções como transporte médico, de policiais e de resgate de corpo de bombeiro em áreas acidentadas topograficamente (GARCIA, CASARRUBIAS, 2011; GRAÇA, et al, 2017).

As aeronaves de asas fixas são indicadas para distâncias superiores de 400 km. Consideram-se os Learjets, turboélices e bimotores, cuja escolha será baseada nas características de pista para pouso, decolagem e disponibilidade. Trata-se de veículos capazes de viajar com certas condições de mau tempo e possuem cabine com espaço maior em relação ao helicóptero - se tornando mais apropriados para técnicas de monitorização e tratamento. A desvantagem está ao necessitar de quatro transferências: transferir o paciente para ambulância, ambulância para o avião, avião para ambulância e ambulância para o hospital de destino (TRAIBER, ANDREOLIO, LUCHESE, 2006). 
Algumas vantagens dos transportes realizados em helicóptero quando comparado aos demais são: meio de transporte rápido; alcança áreas inacessíveis ou difíceis. As desvantagens: espaço limitado para execução de procedimentos; caso o hospital não possua heliporto será necessário um veículo terrestre para complemento do transporte; barulho e vibrações interferem na monitorização e atendimento; cabine não pressurizada; manutenção possui custo elevado; há limites para condições climáticas e necessita pousar para execução de procedimentos no doente 4 . Estudos descrevem que o transporte de neonatos em asa rotativa fornece risco e eventos de sangramentos intracranianos. Por isso esse meio deve ser evitado ou até contraindicado para essa faixa etária (SAÚDE, 2002; SKEOCH, 2005; BOUCHUT, 2011; SHIPLEY, et al, 2019; Bailey, et al, 2018).

Para transportes em aviões, as vantagens são: meio rápido para percorrer longas distâncias; mesmo com mau tempo pode se trafegar; possui pressurização de cabine; cabine com tamanho que permite um melhor atendimento e monitorização. Desvantagens: por necessitar de pista de pouso e decolagem, implica, em outro ponto, quatro transferências (hospital para ambulância / ambulância para avião / avião para ambulância / ambulância para hospital) e também não pode se parar para realização de técnicas (TRAIBER, ANDREOLIO, LUCHESE, 2006).

Para qualquer aeronave escolhida é necessária uma comunicação clara entre equipe médica e pilotos para melhor estabilidade do paciente. Deve-se reunir os comandantes da aeronave juntamente com enfermeiro e médico para elaborar um plano de voo e evitar iatrogenias (TRAIBER, ANDREOLIO, LUCHESE, 2006).

\section{CONSIDERAÇÕES FINAIS}

Pelo que foi dito, é necessário avaliar as condições do meio de transporte a ser utilizado considerando os materiais necessários para realizar a transferência com segurança de todos tripulantes. O transporte deve ser feito sem causar dano a ninguém, possibilitando uma manutenção estável do quadro hemodinâmico do paciente para permitir uma probabilidade maior de cura ao levá-lo para outra instituição buscando reduzir as chances de intercorrências que pudessem colocar em risco a vida não só do paciente como da equipe também. 


\section{REFERÊNCIAS}

Bouchut, Jean \& Van Lancker, Eric \& Chritin, Vincent \& Gueugniaud, Pierre-Yves. (2011). Physical Stressors during Neonatal Transport: Helicopter Compared with Ground Ambulance. Air medical journal. 30. 134-9. 10.1016/j.amj.2010.11.001.

GRACA, Andreia Catarina Gonçalves da et al. Transporte inter-hospitalar do doente crítico: a realidade de um hospital do nordeste de Portugal. Rev. Enf. Ref., Coimbra, v. serlV, n. 15, p. 133-144, dez. 2017.

JÚNIOR, G. A. P.; NUNES, T. L.; FILHO, A. B. Transporte do Paciente Crítico. Medicina, Ribeirão Preto, 34. 134-153, abr/jun. 2017.

LACERDA, M. A.; CRUVINEL, M. G. C.; SILVA, W. V. Transporte de Pacientes: Intra-Hospitalar e Inter-hospitalar.

MANNARINO, L.; TIMERMAN, S. Transporte terrestre e aéreo do paciente crítico. Rev Soc Cadriol de São Paulo, 1998.

MARTÍNEZ, E. C.; TERÁN, B. B.; GARCÍA, J. A. G.; CASARRUBIAS, H. L.; MARTíNEZ, M. M.; JARERO, F. S.; RUBIEL, D. V. Transporte Del Paciente Crítico em Unidades Móviles Terrestres. Revista de La Associación Mexicana de Medicina Critica Y Terapia Intensiva, Cidade do México. 2011.

Portaria 2048/GM do disponível em <http://dtr2001.saude.gov.br/sas/PORTARIAS/Port2002/Gm/GM-2048.htm> acessado em julho de 2019.

Shipley, Lara \& Gyorkos, Timea \& Dorling, Jon \& J Tata, Laila \& Szatkowski, Lisa \& Sharkey, Don. (2019). Risk of Severe Intraventricular Hemorrhage in the First Week of Life in Preterm Infants Transported Before 72 Hours of Age. Pediatric Critical Care Medicine. 20. 638-644. 10.1097/PCC.0000000000001937.

Skeoch $\mathrm{CH}$, Jackson $\mathrm{L}$, Wilson $\mathrm{AM}$, et alFit to fly: practical challenges in neonatal transfers by air Archives of Disease in Childhood - Fetal and Neonatal Edition 2005;90:F456-F460.

Bailey, Vickie \& Szyld, Edgardo \& Cagle, Kristi \& Kurtz, Deborah \& Chaaban, Hala \& Wu, Dee \& Williams, Patricia. (2018). Modern Neonatal Transport: Sound and Vibration Levels and Their Impact on Physiological Stability. American Journal of Perinatology. 36. 10.1055/s-0038-1668171.

TRAIBER, C.; ANDREOLIO, C.; LUCHESE, S. Transporte inter-hospitalar de crianças criticamente doentes. Porto Alegre, PUCRS, 2006. 